Olaf Breidbach/Paul Ziche (Hg.)

Naturwissenschaften um 1800 


\section{Naturwissenschaften um 1800}

Wissenschaftskultur in Jena-Weimar

Herausgegeben von

Olaf Breidbach und Paul Ziche

2001

Verlag Hermann Böhlaus Nachfolger Weimar 
Die Deutsche Bibliothek - CIP-Einheitsaufnahme

Naturwissenschaften um 1800 : Wissenschaftskultur in Jena-Weimar / hrsg. von

Olaf Breidbach und Paul Ziche. - Weimar : Verl. Hermann Böhlaus Nachf., 2001

ISBN 978-3-7400-1177-2

ISBN 978-3-7400-1177-2

ISBN 978-3-476-02828-0 (cBook)

DOI 10.1007/978-3-476-02828-0

Alle Rechte vorbehalten. Ohne schriftliche Genehmigung des Verlages ist es nicht gestattet, das Werk unter Verwendung mechanischer, elektronischer und anderer Systeme in irgendeiner Weise zu verarbeiten und zu verbreiten. Insbesondere vorbehalten sind die Rechte der Vervielfältigung - auch von Teilen des Werkes - auf photomechanischem oder ähnlichem Wege, der tontechnischen Wiedergabe, des Vortrages, der Funk- und Fernsehsendung, der Speicherung in Datenverarbeitungsanlagen, der Übersetzung und der literarischen oder anderweitigen Bearbeitung.

(C) 2001 by Springer-Verlag GmbH Deutschland

Ursprünglich erschienen bei Verlag Hermann Böhlaus Nachfolger Weimar 2001

www.boehlausnf.de

info@boehlausnf.de 


\section{Inhaltsverzeichnis}

Olaf Breidbach und Paul Ziche

Einführung. Naturwissen und Naturwissenschaften - Zur Wissenschaftskultur

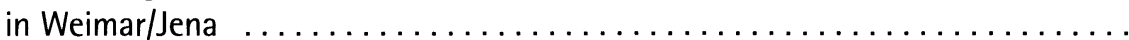

\section{Naturphilosophie und Wissenschaften}

\section{Gian Franco Frigo}

"Der stete und feste Gang der Natur zur Organisation"

Von der Naturgeschichte zur Naturphilosophie um 1800

Olaf Breidbach

Transformation statt Reihung - Naturdetail und Naturganzes

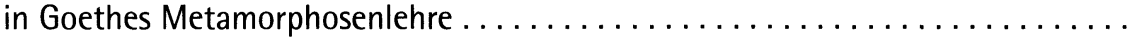

Thomas Bach

„Für wen das hier gesagte nicht gesagt ist, der wird es nicht für überflüssig halten.”

Franz Joseph Schelvers Beitrag zur Naturphilosophie um $1800 \ldots \ldots \ldots \ldots \ldots$. . . .

Matthias John

Carl Christian Erhard Schmid und die Naturwissenschaften

Paul Ziche

Anthropologie zwischen Physiologie und Naturphilosophie.

Wissenschaftssystematische Aspekte der Fachgebiete Anthropologie

und Psychologie um 1800

Temilo van Zantwijk

Anthropologische Aspekte der ,philosophischen Konstruktion'

der ,Naturwissenschaft' bei Schelling

\section{Strukturen wissenschaftlicher Gesellschaften}

Jürgen Kiefer

Sozialgeschichtliche Untersuchungen über die Mitglieder der deutschen Akademien der Wissenschaften am Ende des 18. Jahrhunderts 
Joachim Bauer und Gerhard Müller

Zwischen Theologie und praktischen Wissenschaften:

Der Aufklärer Joachim Georg Darjes

Holger Zaunstöck

Untersuchungen zur Struktur Naturforschender Gesellschaften im 18. Jahrhundert:

Die Sozietäten in Halle, Leipzig und Jena

\section{Rezeption der Jenaer Naturforschung}

\section{Georg Eckardt}

Anspruch und Wirklichkeit der Erfahrungsseelenkunde, dargestellt an Hand periodisch erscheinender Publikationen um 1800

Kai Torsten Kanz

„... man weiß nur was man einem Manne schreiben soll mit dem man einmal persönlich verhandelt hat."

Zum Briefwechsel Goethes mit Christian Gottfried Nees von Esenbeck

Heiko Weber

J. W. Ritter und J. Webers Zeitschrift "Der Galvanismus"

Bernd Klengel

Ultraviolette Strahlen und Ladungssäule. Zur Rezeption von Entdeckungen

Johann Wilhelm Ritters in Frankreich

Gesamtbibliographie

Veröffentlichungen des SFB 482

Register 